Internist 2020 $61: 1010-1016$ https://doi.org/10.1007/s00108-020-00862-5 Online publiziert: 8 . September 2020

(c) Der/die Autor(en) 2020

\section{Redaktion}

B. Salzberger, Regensburg

T. Welte, Hannover

\author{
B. Seeliger $\cdot$ K. Stahl ${ }^{2} \cdot$ Sascha David ${ }^{3,4}$ \\ ${ }^{1}$ Klinik für Pneumologie, Medizinische Hochschule Hannover, Hannover, Deutschland \\ ${ }^{2}$ Klinik für Gastroenterologie, Hepatologie und Endokrinologie, Medizinische Hochschule Hannover, \\ Hannover, Deutschland \\ ${ }^{3}$ Klinik für Nieren- und Hochdruckerkrankungen, Medizinische Hochschule Hannover, Hannover, \\ Deutschland \\ ${ }^{4}$ Institut für Intensivmedizin, Universitätsspital Zürich, Zürich, Schweiz
}

\title{
Extrakorporale Blutreinigungsverfahren in der Sepsis - Update
}

\begin{abstract}
Die Sepsis ist eine lebensbedrohliche Organdysfunktion infolge einer fehlregulierten Wirtsantwort auf eine Infektion mit enormen gesundheitlichen und ökonomischen Folgen [1]. Auch in neueren Untersuchungen bleibt die Mortalität mit bis zu $58 \%$ beträchtlich [2]. Immunmodulatorische Ansätze in der Behandlung der Sepsis sind naheliegend, hierzu gehören auch Strategien, die zumindest eine Reduktion proinflammatorisch wirksamer Zytokine anstreben [3].

\section{》) Die Heterogenität von Erregern und Immunantworten erschwert die Durchführung klinischer Studien}

In der Evaluation solcher Ansätze erschwert die Heterogenität von Erregern und Immunantworten die Durchführung klinischer Studien [4]. In diesem Übersichtsbeitrag beschreiben wir die aktuelle Studienlage zu extrakorporalen Blutreinigungsverfahren in der Sepsis (• Tab. 1).

\section{High-volume-Hämofiltration und Very-high-volume- Hämofiltration}

Das wohl älteste Verfahren zur extrakorporalen Entfernung von schädigenden Stoffen mit kleinem Molekulargewicht ist die kontinuierliche Hämodia- lyse bzw. -filtration unter Einsatz hoher Filtrationsvolumina. Dies lässt sich mit allen gängigen Dialysegeräten auf Intensivstationen durchführen, es bedarf lediglich eines sogenannten High-flux-Filters unter hohen Ultrafiltrations- bzw. Substitutionsvolumina. Zuletzt hat sich die Definition von $35 \mathrm{ml} / \mathrm{kg}$ pro h für die High-volume-Hämofiltration (HV$\mathrm{HF}$ ) sowie $>45 \mathrm{ml} / \mathrm{kg}$ pro $\mathrm{h}$ für die Very-high-volume-Hämofiltration etabliert [5], bisherige Studien waren bezüglich der tatsächlich verabreichten Dosis jedoch sehr variabel.

Bis 2013 waren die meisten Studien zur HVHF kleinere Beobachtungsund teilweise randomisierte Studien. Im Jahr 2013 wurden dann die Ergebnisse der IVOIRE-Studie, der bislang einzigen multizentrischen randomisierten Studie veröffentlicht [6]. Hier wurden eine HFHV mit $70 \mathrm{ml} / \mathrm{kg}$ pro $\mathrm{h}$ und eine Standardhämofiltration mit $35 \mathrm{ml} / \mathrm{kg}$ pro $\mathrm{h}$ bei Patienten mit septischem Schock verglichen. Weder bei der 28-Tage-Mortalität noch in Bezug auf hämodynamische Surrogatparameter war ein signifikanter Unterschied zwischen den Verfahren vorhanden.

Eine Metaanalyse unter Einschluss von insgesamt 4 Studien mit 205 Patienten zeigte ebenfalls keinen signifikanten Unterschied in der Mortalität: relatives Risiko für Mortalität von 0,89 (95\%-Konfidenzintervall 0,6-1,32; [7]). Hierbei ist wichtig, dass es bei den verglichenen Studien eine erhebliche technische Varianz sowohl im Blutfluss (40-95 ml/kg pro h) als auch in der Durchführung (beispielsweise kontinuierlich vs. intermittierend) gab [3]. Obwohl einige Studien in Subgruppenanalysen, wie etwa in Bezug auf den septischen Schock mit akutem Nierenversagen (ANV) nach Verbrennungstrauma, einen Überlebensvorteil durch HVHF zeigten, konnten diese Beobachtungen in systematischen Analysen und der multizentrischen IVOIREStudie nicht bestätigt werden $[8,9]$.

\section{High-cut-off-Filter}

Bei den High-cut-off(HCO)-Filtern handelt es sich um Dialysefilter mit erhöhter Porengröße $(20 \mathrm{~nm})$, die sowohl bei der Sepsis als auch in anderen Situationen, wie bei akutem Nierenschaden im Rahmen der Rhabdomyolyse oder auch bei der Cast-Nephropathie des multiplen Myeloms, eingesetzt werden.

\section{》) Ein positiver Effekt von HCO- Filtern in der Sepsis abseits etablierter Indikationen ist nicht belegt}

Insgesamt wurde für den Einsatz von HCO-Filtern in den initialen Pilotstudien bei Patienten mit sepsisinduzierter akuter Nierenschädigung hauptsächlich eine effektivere Clearance von proinflammatorischen Zytokinen wie Inter- 
Tab. 1 Zusammenfassung der Endpunkte bisheriger Studien zu extrakorporalen Verfahren in der Sepsis

\begin{tabular}{|c|c|c|c|c|c|}
\hline Verfahren & Referenz & $\begin{array}{l}\text { Reduktion } \\
\text { Zytokin- } \\
\text { spiegel }\end{array}$ & $\begin{array}{l}\text { Reduktion Katechola- } \\
\text { minbedarf/Verbesserung } \\
\text { Hämodynamik }\end{array}$ & Mortalitätsreduktion & Kommentar \\
\hline \multicolumn{6}{|l|}{ HVHF } \\
\hline Beobachtungsstudien $^{\mathrm{a}}$ & [32-36] & N.u. & + & (+) (vs. erwartet) & N.u. \\
\hline $\begin{array}{l}\text { RCT, monozentrisch, } \\
\text { „open label" }\end{array}$ & {$[37,38]$} & N.u. & + & Kein Unterschied & N.u. \\
\hline $\mathrm{RCT}$, multizentrisch & {$[6,8]$} & N.u. & $(+)$ & Kein Unterschied & N.u. \\
\hline \multicolumn{6}{|l|}{ HCO Filter } \\
\hline Beobachtungsstudien $^{\mathrm{a}}$ & [39-42] & + & $+[41]$ & N.u. & N.u. \\
\hline $\begin{array}{l}\text { RCT, monozentrisch, } \\
\text { „open label" }\end{array}$ & [10] & + & + & N.u. & N.u. \\
\hline $\mathrm{RCT}$, multizentrisch & [43] & N.u. & Kein Unterschied & Kein Unterschied & Vorzeitig abgebrochen \\
\hline \multicolumn{6}{|l|}{ Adsorptionsverfahren } \\
\hline \multicolumn{6}{|c|}{ Polymyxin-B-Hämoperfusion } \\
\hline $\begin{array}{l}\text { RCT, multizentrisch, } \\
\text { „open label", Pilotstudie }\end{array}$ & {$[11,12]$} & N.u. & + & $+[12]$ & N.u. \\
\hline $\begin{array}{l}\text { RCT, multizentrisch, } \\
\text { "open label" }\end{array}$ & [13] & N.u. & Kein Unterschied & Kein Unterschied & N.u. \\
\hline \multirow[t]{2}{*}{$\begin{array}{l}\text { Retrospektiv, } \\
\text { "propensity score } \\
\text { matching“ }\end{array}$} & \multirow[t]{2}{*}[44,45]{} & & & $\begin{array}{l}\text { Kein Unterschied bei } \\
\text { abdomineller Sepsis } \\
{[44]}\end{array}$ & \\
\hline & & & & $\begin{array}{l}\text { Reduktion bei septi- } \\
\text { schem Schock mit ANV } \\
{[45]}\end{array}$ & \\
\hline $\begin{array}{l}\text { RCT, multizentrisch, } \\
\text { verblindet (EUPHRATES) }\end{array}$ & [14] & N.u. & N.u. & Kein Unterschied & $\begin{array}{l}\text { Mortalitätsreduktion in Sub- } \\
\text { gruppe mit moderat erhöhter } \\
\text { Endotoxinaktivität [15] }\end{array}$ \\
\hline \multicolumn{6}{|c|}{ CytoSorb $^{\circledR}$ (CytoSorbents Europe, Berlin, Deutschland) } \\
\hline Beobachtungsstudien $^{\mathrm{a}}$ & [17] & + & + & N.u. & N.u. \\
\hline $\begin{array}{l}\text { RCT, monozentrisch, } \\
\text { "open label" }\end{array}$ & [18] & + & + & N.u. & N.u. \\
\hline $\begin{array}{l}\text { RCT, multizentrisch, } \\
\text { "open label" }\end{array}$ & {$[16,20]$} & $-[20] /+[16]$ & N.u. & $\begin{array}{l}\text { Kein Unterschied [16, } \\
\text { 20] }\end{array}$ & $\begin{array}{l}\text { Zytokinreduktion bei Sepsis mit } \\
\text { ALI [16] }\end{array}$ \\
\hline $\begin{array}{l}\text { Retrospektiv, "propensi- } \\
\text { ty score matching" }\end{array}$ & [19] & N.u. & N.u. & (+) vs. erwartet & N.u. \\
\hline \multicolumn{6}{|l|}{ CPFA } \\
\hline \multirow[t]{2}{*}{$\begin{array}{l}\text { RCT, multizentrisch, } \\
\text { "open label“ }\end{array}$} & \multirow[t]{2}{*}[26,27]{} & & \multirow[t]{2}{*}{-} & $\begin{array}{l}\text { Erhöhte } 72 \text { h-Mortalität } \\
\text { [27] }\end{array}$ & \multirow{2}{*}{$\begin{array}{l}\text { ROMPA-Studie vorzeitig beendet } \\
\text { nach negativen Ergebnissen der } \\
\text { COMPACT-2-Interimsanalyse }\end{array}$} \\
\hline & & & & Kein Unterschied [26] & \\
\hline \multicolumn{6}{|l|}{ TPE } \\
\hline Beobachtungsstudien $^{\mathrm{a}}$ & {$[31]$} & + & + & N.u. & N.u. \\
\hline $\mathrm{RCT}$, monozentrisch & {$[46]$} & N.u. & N.u. & + & N.u. \\
\hline $\mathrm{RCT}$, multizentrisch & NCT04231994 & N.u. & N.u. & N.u. & Rekrutierungsphase \\
\hline
\end{tabular}

leukin(IL)-6, IL-10, Tumor-NekroseFaktor a (TNF- $\alpha)$ und Interleukin-1Rezeptor-Antagonist (IL-1ra) im Vergleich zu klassischen High-flux-Filtern gezeigt. Eine kleine monozentrische randomisierte Studie mit 30 Patienten konnte zudem eine Reduktion der Katecholamindosis gegenüber High-fluxFiltern zeigen [10]. Eine retrospektive Beobachtungsstudie mit 16 Patienten ließ erstmals einen positiven Effekt der Methode auf die Mortalität vermuten
(Mortalität 37,5\% mit HCO-Filter vs. $87,5 \%$ mit kontinuierlicher venovenöser Hämodiafiltration, $p=0,03)$. Die randomisierte High Cut-Off Sepsis Study (HICOSS) zur Überprüfung dieser Resultate wurde allerdings abgebrochen, 
als in einer Interimsanalyse weder ein Vorteil in der 28-Tage-Mortalität noch eine Reduktion des Katecholamineinsatzes gezeigt werden konnte. Insgesamt gibt es deshalb keine Evidenz für einen positiven Effekt von HCO-Filtern in der Sepsis abseits etablierter Indikationen wie der Rhabdomyolyse.

\section{Adsorptionsverfahren}

\section{Polymyxin-B-Hämoperfusion}

Endotoxin gehört $\mathrm{zu}$ den sogenannten „pathogen-associated molecular patterns" (PAMP) und eine hohe Serumaktivität, die mittels „endotoxin activity assay“ (EAA) einfach bestimmt werden kann, ist mit erhöhter Mortalität assoziiert. Mittels Hämoperfusion über eine Polymyxin-B(PMX)-Kartusche lässt sich eine effektive Elimination des Endotoxins erwirken (• Abb. 1). Dieses Verfahren wurde erstmals bei Patienten mit abdomineller Sepsis eingesetzt [11]. In einer Pilotstudie konnte eine Reduktion des Katecholaminbedarfs gezeigt werden und in der späteren EUPHASStudie [12] auch der Mortalität. Um die Heterogenität des Patientenkollektivs zu reduzieren und die Wahrscheinlichkeit des Vorhandenseins einer hohen Endotoxinkonzentration zu erhöhen, wurden im Sinne eines "predictive enrichment“ ausschließlich Patienten mit abdomineller Sepsis eingeschlossen.

In der 2015 veröffentlichten ABDOMIX-Studie konnten diese positiven Effekte allerdings nicht komplett bestätigt werden [13]. Als möglicher Grund wurde ein häufiges „clotting“ der PMX-Kartuschen angeführt, womit nur $70 \%$ der Kohorte die avisierten 2 Behandlungen à $2 \mathrm{~h}$ erhielten. Die 2018 veröffentlichte EUPHRATES-Studie als bislang methodisch höchstwertige multizentrische Studie zum Einsatz von PMX im septischen Schock zeigte für die 28-Tage-Mortalität als primären Endpunkt keinen positiven Effekt der Methode [14]. Schlüsselpunkt der Studie war eine Randomisierung und Stratifizierung in Abhängigkeit von der Höhe der Endotoxinämie, sodass nur Patienten mit erhöhtem Endotoxinspiegel eingeschlossen wurden. Prinzipiell ist dies geeignet, um die Heterogeni-

Internist 2020 $\cdot 61: 1010-1016$ https://doi.org/10.1007/s00108-020-00862-5

(c) Der/die Autor(en) 2020

\section{B. Seeliger · K. Stahl·S. David}

Extrakorporale Blutreinigungsverfahren in der Sepsis - Update

\section{Zusammenfassung}

Hintergrund. Trotz fortwährender Entwicklungen bleibt die Mortalität in der Sepsis beträchtlich. Verschiedene Verfahren zur extrakorporalen Zytokinelimination wurden beschrieben, aber die Datenlage ist widersprüchlich.

Ziel der Arbeit. Diese Arbeit soll eine Übersicht über aktuelle extrakorporale Blutreinigungsverfahren und deren Studienlage geben.

Methoden. Nichtsystematischer Literaturreview

Ergebnisse. Aktuell gibt es verschiedene Blutreinigungsverfahren mit unterschiedlicher Evidenz bezüglich der Reduktion von proinflammatorischen Zytokinen, Katecholaminbedarf und Mortalität, darunter die sogenannte High-volume-Hämofiltration mit und ohne High-cut-off-Filter sowie spezielle Adsorber (unter anderem CytoSorb ${ }^{\circledR}$ [CytoSorbents Europe, Berlin, Deutschland] und Polymyxin B). An Bedeutung gewinnt aktuell auch wieder der therapeutische Plasmaaustausch. Bei einigen Verfahren mit initial vielversprechenden Ergebnissen darunter die kombinierte Plasmafiltration und -adsorption - zeigten multizentrische randomisierte Studien jedoch sogar einen negativen Effekt.

Schlussfolgerung. Die theoretische Begründung ist naheliegend, obwohl die aktuelle Evidenz keine Empfehlung auf Leitlinienqualität erlaubt. Die Heterogenität der Immunantwort in der Sepsis erschwert die Durchführung multizentrischer randomisierter Studien, die initial positive Effekte bislang oft nicht bestätigen konnten. Neue Studien zu den Blutreinigungsverfahren benötigen daher eine klar definierte Subgruppe des septischen Schocks. Entsprechend sollten die Verfahren nicht als konkurrierend, sondern im Sinne einer individualisierten Therapie komplementär eingesetzt werden.

\section{Schlüsselwörter}

Plasmapherese - Septischer Schock · Hämodialyse $\cdot$ Hämofiltration $\cdot$ Proinflammatorische Zytokine

\section{Extracorporeal techniques for blood purification in sepsis: an update}

Abstract

Background. Despite ongoing development, mortality in sepsis remains considerable. Various techniques for extracorporeal cytokine removal have been described, but evidence remains conflicting.

Objectives. The aim of this article is to summarize currently used extracorporeal blood purification techniques and their evidence.

Methods. Non-systematic literature review Results. There are currently various blood purification techniques used with different levels of evidence regarding cytokine removal, vasopressor sparing effects and reduction of mortality, including high-volume dialysis with and without high cut-off filters, special adsorption filters (including CytoSorb ${ }^{\circledR}$, CytoSorbents Europe, Berlin, Germany, and polymyxin-B filters). There is development regarding therapeutic plasma exchange. For some blood purification techniques such as combined plasma filtration and adsorption, multicentric randomized studies found a negative effect on survival.

Conclusions. Despite a theoretical rationale, the use of blood purification methods cannot be recommended for sepsis patients due to the lack of evidence of their efficacy. Heterogeneous inflammatory responses in sepsis render conduction of larger trials difficult. Thus, future studies should cautiously identify appropriate sepsis subtypes to be included. Available techniques should be chosen as individualized complementary treatments and not as competing systems.

Keywords

Plasma exchange $\cdot$ Shock, septic $\cdot$ Hemodialysis · Hemofiltration - Cytokines, proinflammatory 




Abb. $1 \Delta$ Übersicht von durch Adsorptionsverfahren eliminierten Botenstoffen, Substraten und zellulären Bestandteilen. Dunkelblau Bestandteile, die mutmaßlich einen pathogenen Einfluss in der Sepsis haben und deren Elimination somit einen potenziellen Nutzen verspricht. Hellblau Per se nützliche Stoffe, durch deren Elimination wiederum potenziell negative Effekte möglich sind. ALT Alanin-Aminotransferase, AST Aspartat-Aminotransferase, CXCL-1 "chemokine (C-X-C motif) ligand 1", $D A M P$ "damage-associated molecular patterns", FGF "fibroblast growth factor", G-CSF granulozytenkoloniestimulierender Faktor, GM-CSF Granulozyten-Makrophagen-Kolonie-stimulierender Faktor, HMGB1 "high mobility group box 1 protein",

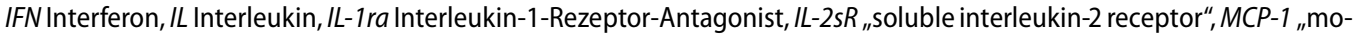
nocyte chemoattractant protein 1", MIP "macrophage inflammatory protein", PAI-1 Plasminogenaktivatorinhibitor Typ 1, $P A M P$ "pathogen-associated molecular patterns", $P C T$ Prokalzitonin, sUPAR "soluble urokinase plasminogen activator receptor", T3 Trijodthyronin, TNF- $a$ Tumor-Nekrose-Faktor a, TSST-1 "toxic shock syndrome toxin 1"

tät zu reduzieren, allerdings zeigte eine Nachanalyse, dass die theoretische Endotoxinclearance von PMX bei sehr hohen EAA-Werten teilweise deutlich überschritten wurde. In einer Post-hoc-Analyse wurde daher eine Subgruppenanalyse nach Endotoxinaktivität durchgeführt, die für mäßig erhöhte Endotoxinaktivitätswerte eine signifikante Verbesserung der Hämodynamik und sogar der Prognose zeigte [15]. Diese Ergebnisse bedürfen jedoch einer prospektiven Validierung.

\section{CytoSorb $^{\circledR}$}

Der CytoSorb ${ }^{\circledR}$-Adsorber (CytoSorbents Europe, Berlin, Deutschland) besteht aus kleinen geladenen Kügelchen mit einer sehr großen Oberfläche $\left(40.000 \mathrm{~m}^{2}\right)$ : $\mathrm{Er}$ kann zahlreiche Zytokine, Chemokine, PAMP und „damage-associated molecular patterns" (DAMP), aber keine Endotoxine aus dem Vollblut durch simple Hämoperfusion adsorbieren.

\section{》) Ein klinischer Nutzen des CytoSorb $^{\circledR}$-Adsorbers ist bisher nicht hinreichend nachgewiesen}

Daten zu diesem eher neueren Hämoadsorptionsfilter sind bisher wenig vorhanden. In einer initialen Multicenterstudie zeigte sich eine Reduktion der IL-6-Konzentrationen nach CytoSorb ${ }^{\circledR}$ Anwendung. Eine Reduktion der Mortalität konnte nicht gezeigt werden, allerdings war die Größe der Studie hierzu nicht geeignet [16]. In einer prospekti- ven Open-label-Studie unter Einschluss von 20 Patienten mit therapierefraktärem septischem Schock konnte eine Reduktion von Laktat und des Katecholaminbedarfs gezeigt werden [17]. Diese Ergebnisse wurden in einer randomisierten Studie bestätigt, in der zusätzlich ein signifikanter Abfall von Prokalzitonin beobachtet wurde [18]. Eine aktuelle retrospektive Auswertung von 67 Patienten mit septischen Schock, die mit Dialyse plus CytoSorb ${ }^{\circledR}$-Filter behandelt wurden, ergab eine Mortalitätsreduktion im Vergleich zu einer mittels ,propensity score matching" ausgewählten Kontrollkohorte ohne CytoSorb ${ }^{\circledR}$-Filter. Als Referenz wurde dabei die erwartete Mortalität nach Sequential-Organ-Failure-Assessment(SOFA)-Score herangezogen [19]. Die Adsorptionskapazität für IL-6 konnte 
in einer späteren randomisierten Studie bestätigt werden, allerdings fand sich weder ein Abfall des zirkulierenden IL-6 noch eine Reduktion der Sterblichkeit [20].

Trotz zahlreicher Einzelfallberichte mit vermeintlich positiver Beeinflussung des Krankheitsverlaufs ist ein klinischer Nutzen bisher nicht hinreichend nachgewiesen. Vorläufige Daten der Auswertung der Cytoscore-Studie (NCT03977688) zeigten für die sehr frühe Anwendung bei sehr kranken Patienten unter Einbeziehung des neu validierten „CytoSorbScores“, der möglicherweise in zukünftigen Studien eine Hilfestellung bei der Stratifizierung bieten wird, einen Hinweis auf eine niedrigere Mortalität [21].

\section{Weitere Adsorptionsverfahren}

Eine Vielzahl weiterer Adsorptionsfilter wird derzeit evaluiert. In zwei monozentrischen randomisierten Studien zum HA-330-Adsorptionsfilter zeigten sich ein Abfall von Inflammationsmarkern und Verbesserungen der Hämodynamik [22] sowie der Beatmungsintensität und -dauer [23].

Der AN69-basierte Oxiris ${ }^{\circledR}$-Filter (Baxter, Illinois, USA) enthält eine positiv geladene Polyethylenaminschicht, die durch einen dreischichtigen Aufbau neben Zytokinen auch negativ geladenes Endotoxin binden kann. In retrospektiven Studien konnte eine deutliche Katecholamineinsparung beobachtet werden [24]. In einer an 2 Zentren durchgeführten randomisierten, kontrollierten Studie wurde der Adsorber bei durch eine Infektion mit gramnegativen Erregern bedingtem septischem Schock und ANV angewendet, wobei eine Reduktion inflammatorischer Zytokine sowie der Katecholamindosis berichtet wurde [25]. Derzeit liegen aber noch keine Daten aus randomisierten Studien vor.

\section{Kombinierte Plasmafiltration und -adsorption}

Das Konzept der kombinierten Plasmafiltration und -adsorption (CPFA) besteht in der initialen Trennung von Plasma und zellulären Blutbestandteilen, wo- durch das separierte Plasma durch den Adsorptionsfilter zur Bindung diverser Zytokine geleitet wird und anschließend wieder den separierten zellulären Blutbestandteilen zugeführt wird.

Inzwischen liegen ernüchternde Ergebnisse aus drei multizentrischen randomisierten Studie vor: COMPACT-1 wurde nach Randomisierung von 192 der 330 geplanten Patienten abgebrochen, da sich keine klinisch relevanten Effekte zeigten [26]. Die Nachfolgestudie COMPACT-2 (NCT01639664) wurde aufgrund erhöhter Mortalität in der Filtrationsgruppe nach der planmäßigen Interimsanalyse abgebrochen. Dies führte auch zur vorzeitigen Beendigung der ROMPA-Studie [27], wodurch es unwahrscheinlich ist, dass die CPFA in der Sepsis in Zukunft Anwendung finden wird.

\section{Therapeutischer Plasma- austausch}

Der therapeutische Plasmaaustausch (TPE) stellt eine infrastrukturell etwas aufwendigere Behandlungsmethode dar. Das Verfahren an sich ist Nephrologen und Hämatologen sehr gut bekannt und gehört beispielsweise zur Standardtherapie der thrombotisch-thrombozytopenischen Purpura (TTP). Die theoretische Begründung für diese Behandlung in der Sepsis stützt sich auf zwei fundamentale Grundprinzipien: Einerseits ermöglicht sie eine hocheffektive Elimination schädigender zirkulierender Moleküle analog zu den zuvor beschriebenen Adsorptionsverfahren innerhalb kurzer Zeit (Prozedurdauer etwa $120 \mathrm{~min}$ ). Andererseits erfolgt ein Ersatz verbrauchter protektiver Plasmafaktoren, beispielsweise von Antipermeabilitätsfaktoren wie Angiopoetin-1, ADAMTS13 und Protein C, die in gefrorenem Frischplasma („fresh frozen plasma“ [FFP]) in hoher Konzentration enthalten sind ([28]; - Abb. 2).

Ein Mangel an ADAMTS13, dem pathophysiologischen Korrelat der TTP, führt dazu, dass der vom Endothel ausgeschüttete von-Willebrand-Faktor (vWF) nicht durch ADAMTS13 in kleine Fragmente gespalten werden kann und somit eine vermehrte Aktivierung und Ag- gregation von Thrombozyten in den Kapillaren bewirkt. Hierdurch kommt es wiederum zur Stase der Mikrozirkulation mit entsprechender Beeinträchtigung des Metabolismus in betroffenen Organen [29]. ADAMTS13 ist auch im septischen Schock stark vermindert, sodass eine Substitution durch einen TPE wie auch die Substitution der Antipermeabilitätsfaktoren Protein C und Angiopoetin-1 ein interessanter Ansatz ist (unveröffentlichte eigene Daten).

\section{) Die Substitution von ADAMTS13 im septischen Schock ist ein interessanter Ansatz}

Eine Metaanalyse, die 4 Studien mit sehr heterogenem Patientenkollektiv untersuchte, zeigte eine Mortalitätsreduktion bei erwachsenen Patienten um 37\% [30]. Die Heterogenität der Studien in Bezug auf den Schweregrad der Sepsis, die Organdysfunktion und insbesondere auch den Zeitpunkt des TPE seit Erkrankungsbeginn ist allerdings hoch.

Zur Untersuchung von Patienten mit schwerem septischem Schock führten wir an unserem Zentrum eine prospektive $\mathrm{Pi}$ lotstudie durch. Nach Einsatz eines TPE im frühen ( $<24 \mathrm{~h}$ nach Diagnosestellung) und schweren septischen Schock (Noradrenalin $>0,4 \mu \mathrm{g} / \mathrm{kg}$ pro min) zeigte sich bei 24 Patienten eine deutliche Verbesserung der Hämodynamik mit Reduktion des Katecholaminbedarfs [31]. Eine prospektive, randomisierte Studie zum Einsatz des TPE bei frühem und besonders schwerem septischem Schock (EXCHANGE; NCT04231994), in der diese Ergebnisse überprüft werden sollen, ist an unserem Zentrum und am Universitätsklinikum Bonn bereits angelaufen. Die Randomisierung wurde August 2020 abgeschlossen und die Studienergebnisse werden derzeit ausgewertet. Eine Ausweitung auf bisher 27 weitere nationale Zentren wird derzeit begutachtet.

\section{Nebenwirkungen extra- korporaler Verfahren}

Alle oben genannte Verfahren bergen neben potenziellen Vorzügen auch Risiken. Allen Verfahren ist das erhöhte Risiko 




Abb. $2 \Delta$ Schematische Übersicht über Substanzklassen, die in der therapeutischen Plasmapherese substituiert (hellblau) und eliminiert (dunkelblau) werden. ADAMTS13 „a disintegrin and metalloprotease with a thrombospondin type 1 motif, member 13", AT-III Antithrombin III, DAMP "damage-associated molecular patterns", IgA Immunglobulin A, IgG Immunglobulin G, IgM Immunglobulin M, FFP "fresh frozen plasma", PAMP "pathogen-associated molecular patterns", ULVWF-M ultralanger von-Willebrand-Faktor-Multimer, VEGF "vascular endothelial growth factor"

einer Thrombosierung der Schlauchsysteme gemeinsam. Ihr Einsatz erfordert oft eine entsprechende Expertise beim Anwender, insbesondere beim Einsatz der regionalen Citratantikoagulation. Eine Filter- bzw. Schlauchthrombosierung kann zu relevanten Thrombozytenabfällen und gegebenenfalls Transfusionen führen. Einige der Verfahren sind durch vermehrten Verlust körpereigener Proteine gekennzeichnet (insbesondere bei High-cut-off-Filtern) oder erhöhen das Risiko von Elektrolytstörungen wie der Hypophosphatämie. Ein weiteres Problem ist die nichtselektive Entfernung anderer essenzieller Substanzen, insbesondere auch von antiinfektiv wirksamen Medikamenten, die nur teilweise durch therapeutisches Drug Monitoring erfasst werden und entsprechend kompensierbar sind.

\section{Korrespondenzadresse}

Prof. Dr. med. Sascha David

Institut für Intensivmedizin, Universitätsspital Zürich

Rämistrasse 100, Zürich, Schweiz

sascha.david@usz.ch

Funding. Open access funding provided by University of Zurich

\section{Einhaltung ethischer Richtlinien}

Interessenkonflikt. B. Seeliger, K. Stahl und S. David geben an, dass kein Interessenkonflikt besteht.

Für diesen Beitrag wurden von den Autoren keine Studien an Menschen oder Tieren durchgeführt. Für die aufgeführten Studien gelten die jeweils dort angegebenen ethischen Richtlinien.

Open Access. Dieser Artikel wird unter der Creative Commons Namensnennung 4.0 International Lizenz veröffentlicht, welche die Nutzung, Vervielfältigung, Bearbeitung, Verbreitung und Wiedergabe in jeglichem Medium und Format erlaubt, sofern Sie den/die ursprünglichen Autor(en) und die Quelle ordnungsgemäß nennen, einen Link zur Creative Commons Lizenz beifügen und angeben, ob Änderungen vorgenommen wurden.

Die in diesem Artikel enthaltenen Bilder und sonstiges Drittmaterial unterliegen ebenfalls der genannten Creative Commons Lizenz, sofern sich aus der Abbildungslegende nichts anderes ergibt. Sofern das betreffende Material nicht unter der genannten Creative Commons Lizenz steht und die betreffende Handlung nicht nach gesetzlichen Vorschriften erlaubt ist, ist für die oben aufgeführten Weiterverwendungen des $\mathrm{Ma}$ terials die Einwilligung des jeweiligen Rechteinhabers einzuholen.

- Es gibt eine Vielzahl an Strategien zur Entfernung potenziell schädlicher Moleküle aus dem Blut septischer Patienten mit teilweise sehr unterschiedlichem methodischem Ansatz.

- Die theoretische Grundlage ist vorhanden, allerdings lassen die bisherigen Ergebnisse zur Wirksamkeit keine klare Empfehlung für den Einsatz einer dieser Methoden zu.

- Der therapeutische Plasmaaustausch beim septischen Patienten substituiert (additiv zu Filtrationseffekten) protektive Proteine. Eine multizentrische randomisierte Studie bei schwerkranken Patienten im septischen Schock ist in Deutschland geplant.
Weitere Details zur Lizenz entnehmen Sie bitte der Lizenzinformation aufhttp://creativecommons.org/ licenses/by/4.0/deed.de.

\section{Literatur}

1. Singer M, Deutschman CS, Seymour CWetal (2016) The third international consensus definitions for sepsis and septic shock (sepsis-3). JAMA 315:801

2. Fleischmann C, Thomas-Rueddel DO, Hartmann M et al (2016) Hospital incidence and mortality rates of sepsis. Dtsch Arztebl Int 113:159

3. Ankawi G, Neri M, Zhang J, Breglia A, Ricci Z, Ronco C (2018) Extracorporeal techniques for the treatment of critically ill patients with sepsis beyond conventional blood purification therapy: the promises and the pitfalls. Crit Care 22:262

4. Seymour CW, Kennedy JN, Wang S et al (2019) Derivation, validation, and potential treatment implications of novel clinical phenotypes for sepsis. JAMA 321:2003

5. Villa G, Neri M, Bellomo Retal (2016) Nomenclature for renal replacement therapy and blood purification techniques in critically ill patients: practical applications. Crit Care 20:283 
6. Joannes-Boyau O, Honoré PM, Perez P et al (2013) High-volume versus standard-volume haemofiltration for septic shock patients with acute kidney injury (IVOIRE study): a multicentre randomized controlled trial. Intensive Care Med 39:1535

7. BorthwickEM, HillCJ, RabindranathKS, Maxwell AP McAuley DF, Blackwood B (2017) High-volume haemofiltration for sepsis in adults. Cochrane Database Syst Rev 1:CD8075

8. Chung KK, Coates EC, Smith DJ et al (2017) Highvolume hemofiltration in adult burn patients with septic shock and acute kidney injury: a multicenter randomized controlled trial. Crit Care 21:289

9. You B, Zhang YL, Luo GX et al (2018) Early application of continuous high-volume haemofiltration can reduce sepsis and improve the prognosis of patients with severe burns. Crit Care 22:173

10. Morgera S, Haase M, Kuss T et al (2006) Pilot study on the effects of high cutoff hemofiltration on the need for norepinephrine in septic patients with acute renal failure. Crit Care Med 34:2099

11. Vincent J-L, Laterre P-F, Cohen J et al (2005) A pilotcontrolled study of a polymyxin B-immobilized hemoperfusion cartridge in patients with severe sepsis secondary to intra-abdominal infection. Shock 23:400

12. Cruz DN, Antonelli M, Fumagalli Ret al (2009) Early use of polymyxin $B$ hemoperfusion in abdominal septic shock: the EUPHAS randomized controlled trial.JAMA 301:2445

13. Payen DM, Guilhot J, Launey Y et al (2015) Early use of polymyxin B hemoperfusion in patients with septic shock due to peritonitis: a multicenter randomized control trial. Intensive Care Med 41:975

14. Dellinger RP, Bagshaw SM, Antonelli M et al (2018) Effect of targeted polymyxin B hemoperfusion on 28-day mortality in patients with septic shock and elevated endotoxin level: the EUPHRATES randomized clinical trial. JAMA 320:1455

15. Klein DJ, Foster D, Walker PM, Bagshaw SM, Mekonnen H, Antonelli M (2018) Polymyxin B hemoperfusion in endotoxemic septic shock patients without extreme endotoxemia: a posthoc analysis of the EUPHRATES trial. Intensive Care Med 44:2205

16. Schädler D, Porzelius C, Jörres A et al (2013) A multicenter randomized controlled study of an extracorporeal cytokine hemoadsorption device in septic patients. Crit Care 17(Suppl 2):P62

17. FrieseckeS, StecherS-S, GrossS, FelixSB, Nierhaus A (2017) Extracorporeal cytokine elimination as rescue therapy in refractory septic shock: a prospective single-center study. J Artif Organs 20:252

18. Hawchar F, László I, Öveges N, Trásy D, Ondrik Z, Molnar Z (2019) Extracorporeal cytokine adsorption in septic shock: a proof of concept randomized, controlled pilot study. J Crit Care 49:172

19. Brouwer WP, Duran S, Kuijper M, Ince C (2019) Hemoadsorption with CytoSorb shows a decreased observed versus expected 28-day all-cause mortality in ICU patients with septic shock: a propensity-score-weighted retrospective study. Crit Care 23:317

20. Schädler D, Pausch C, Heise D et al (2017) The effect of a novel extracorporeal cytokine hemoadsorption device on IL-6 elimination in septic patients: a randomized controlled trial. PLoS ONE 12:e187015

21. Kogelmann K, Hübner T, Schwameis F, Drüner $M$, Scheller M, Jarczak D (2019) Evaluating a Cytosorb Score in septic shock (ECSISS study): 9th Internatio- nal Congress "Sepsis and Multiorgan Dysfunction" Weimar Sepsis Update 2019-Tribute to Translation. Infection 2019:36

22. Huang Z, Wang S-R, Su W, Liu J-Y (2010) Removal of humoral mediators and the effect on the survival of septic patients by hemoperfusion with neutral microporous resin column. Ther Apher Dial 14:596

23. Huang Z, Wang S-R, Yang Z, Liu J-Y (2013) Effect on extrapulmonary sepsis-induced acute lung injury by hemoperfusion with neutral microporous resin column. Ther Apher Dial 17:454

24. Schwindenhammer V, Girardot T, Chaulier K et al (2019) oXiris ${ }^{\circledR}$ use in septic shock: experience of two French centres. Blood Purif 47(Suppl 3):1

25. Broman ME, Hansson F, Vincent J-L, Bodelsson M (2019) Endotoxin and cytokine reducing properties of the oXiris membrane in patients with septic shock: a randomized crossover double-blind study. PLoSONE 14:e220444

26. Livigni S, Bertolini G, Rossi C et al (2014) Efficacy of coupled plasma filtration adsorption (CPFA) in patients with septic shock: a multicenter randomised controlled clinical trial. BMJ Open 4:e3536

27. Giménez-Esparza C, Portillo-Requena C, ColominaCliment F et al (2019) The premature closure of ROMPA clinical trial: mortality reduction in septic shock by plasma adsorption. BMJ Open 9:e30139

28. David S, Stahl K (2019) To remove and replace-a role for plasma exchange in counterbalancing the host response in sepsis. Crit Care 23:14

29. Bockmeyer CL, Claus RA, Budde U et al (2008) Inflammation-associated ADAMTS13 deficiency promotes formation of ultra-large von Willebrand factor. Haematologica 93:137

30. Rimmer E, Houston BL, Kumar A et al (2014) The efficacy and safety of plasma exchange in patients with sepsis and septic shock: a systematic review and meta-analysis. Crit Care 18:699

31. Knaup H, Stahl K, Schmidt BMW et al (2018) Early therapeutic plasma exchange in septic shock: a prospective open-label nonrandomized pilot study focusing on safety, hemodynamics, vascular barrier function, and biologic markers. Crit Care 22:285

32. Honore PM, Jamez J, Wauthier M et al (2000) Prospective evaluation of short-term, high-volume isovolemic hemofiltration on the hemodynamic course and outcome in patients with intractable circulatory failure resulting from septic shock. Crit Care Med 28:3581

33. Joannes-Boyau O, Rapaport S, Bazin R, Fleureau C, Janvier G (2004) Impact of high volume hemofiltration on hemodynamic disturbance and outcome during septic shock. ASAIO J 50:102

34. Ratanarat R, Brendolan A, Piccinni P et al (2005) Pulse high-volume haemofiltration for treatment of severe sepsis: effects on hemodynamics and survival. Crit Care 9:R294-302

35. Cornejo R, Downey P, Castro R et al (2006) Highvolume hemofiltration as salvage therapy in severe hyperdynamic septic shock. Intensive Care Med 32:713

36. Piccinni P, Dan M, Barbacini S et al (2006) Early isovolaemic haemofiltration in oliguric patients with septic shock. Intensive Care Med 32:80

37. Cole L, Bellomo R, Journois D, Davenport $P$, Baldwin I, Tipping P (2001) High-volume haemofiltration in human septic shock. Intensive Care Med 27:978

38. Boussekey N, Chiche A, Faure K et al (2008) A pilot randomized study comparing high and low volume hemofiltration on vasopressor use in septic shock. Intensive Care Med 34:1646
39. Morgera S, Haase M, Rocktäschel J et al (2003) Intermittent high-permeability hemofiltration modulates inflammatory response in septic patients with multiorgan failure. Nephron Clin Pract 94:c75-80

40. Morgera S, Rocktäschel J, Haase M et al (2003) Intermittent high permeability hemofiltration in septic patients with acute renal failure. Intensive Care Med 29:1989

41. Chelazzi C, Villa G, D'Alfonso MG et al (2016) Hemodialysis with high cut-off hemodialyzers in patients with multi-drug resistant gram-negative sepsis and acute kidney injury: a retrospective, case-control study. Blood Purif 42:186

42. Kade G, Lubas A, Rzeszotarska A, Korsak J, Niemczyk S (2016) Effectiveness of high cut-off hemofilters in the removal of selected cytokines in patients during septic shock accompanied by acute kidney injury-preliminary study. Med Sci Monit 22:4338

43. Honore PM, Clark W (2009) Novel therapeutical concepts for extracorporeal treatment of hyperinflammation and sepsis: immunomodulation. approach with a novel high Cut-OFF membrane: the SepteX membrane. In: Proceedings of 10th Congress of World Federation of CCU WFSICCM, Florence

44. Iwagami M, Yasunaga H, Doi K et al (2014) Postoperative polymyxin $B$ hemoperfusion and mortality in patients with abdominal septic shock: a propensity-matched analysis. Crit Care Med 42:1187

45. Iwagami M, Yasunaga $H$, Noiri E et al (2016) Potential survival benefit of polymyxin $B$ hemoperfusion in septic shock patients on continuous renal replacement therapy: a propensity-matched analysis. Blood Purif 42:9

46. Busund R, Koukline V, Utrobin U, Nedashkovsky E (2002) Plasmapheresis in severe sepsis and septic shock: a prospective, randomised, controlled trial. Intensive Care Med 28:1434 